\title{
Human Papilloma Virus, Cellular Genetics and Susceptibility to Cervical Cancer
}

\author{
Sreekala Nair and M Radhakrishna Pillai \\ Department of Molecular Medicine, Drug Development and Chemoinformatics \\ Regional Cancer Centre, Thiruvananthapuram, Kerala, India
}

KEYWORDS Human papillmoa virus; cervical cancer; genetic susceptibility; polymorphisms

\begin{abstract}
Research in relation to the etiology of cervical cancer has made substantial progress in the last two decades both in scientific and operational terms. In many countries, HPV is the most common sexually transmitted infection (STI) and cervical cancer remains the second most common cancer among women worldwide. Although high risk HPV infection has been identified as the primary etiological agent for cervical cancer, various co-factors such as tobacco derived carcinogens, inhalation of air cotaminated through the combustion of coal and kitchen smoke have also been reported to be associated with cervical cancer. Studies on HPV 16 E6 and E7 gene variations provide evidence for the association of specific E6 gene variants with the risk of cervical cancer. While E6 variants may be important in contributing to increased severity of cervical cancer, polymorphisms of other host cellular proteins, such as p53 and p73, may also play an as yet undefined role in modulating the E6-mediated carcinogenic process. Tobacco smoking and chewing has also been found to be associated with increased risk of cervical malignancy. Major classes of carcinogens present in tobacco and tobacco smoke are converted into DNA-reactive metabolites by cytochrome P450 (CYP)-related enzymes, several of which display genetic polymorphism. Individual susceptibility to cancer is likely to be modified by the genotype for enzymes involved in the activation or detoxification of carcinogens in tobacco and repair of DNA damage. Polymorphisms in the carcinogenmetabolizing enzymes are thought to play a role in cancer susceptibility in humans. Associations of polymorphisms with cancer risk will be especially important in cases where there are known exposures to chemical carcinogens such as with tobacco smoking, high intake of food mutagens and industrial exposures. HPV infection, through the modulation of cellular xenobiotic metabolizing enzymes, may play a role in the ability of cells to handle environmental carcinogens.
\end{abstract}

\section{INTRODUCTION}

Research in relation to the etiology of cervical cancer has made substantial progress in the last two decades both in scientific and operational terms. For decades the epidemiological profile of women with cervical cancer was recognized as suggestive of a sexually transmitted process and several infectious agents were proposed over the years including syphilis, gonorrhea, Chlamydia Trachomatis and type 2 Herpes Simplex Virus. The development of technology to detect the presence of Human Papilloma Virus (HPV) DNA in cellular specimens in the early 1980s made possible the establishment of a definite etiological role for HPV in cervical cancer (Bosch et al. 2002). The association of HPV with cervical cancer has provided the background and the scientific justification for improving screening programs and for developing HPV vaccines (Bosch et al. 2002). In many countries, HPV is the most common sexually transmitted infection (STI) and cervical cancer remains the second most common cancer among women worldwide. In countries where screening is not implemented, cervical cancer is still a major health problem and a frequent cause of death (Bosch et al. 2002). In India, carcinoma of the uterine cervix accounts for about $26 \%$ of female cancers, resulting in about 95,000 women developing the disease annually (Jayant et al. 1995). Thus we have $1 / 6^{\text {th }}$ of the world's population and $1 / 3^{\text {rd }}$ of the world's cervical cancer burden. Although high risk HPV infection has been identified as the primary etiological agent for cervical cancer, various cofactors such as tobacco derived carcinogens, inhalation of air contaminated through the combustion of coal and kitchen smoke have also been reported to be associated with cervical cancer. Interindividual genetic differences in susceptibility to various carcinogens are important host factors associated with human cancer. It is thus possible that variations in oncogenes, tumor suppressor genes or xenobiotic metabolizing genes may influence cervical carcinogenesis.

\section{HUMAN PAPILLOMA VIRUS(HPV)}

Papilloma viruses are epitheliotropic viruses present in the skin and mucosa of several animals. 
In humans, more than 70 types have been described (Zur Hausen H 1996). Recognized as sexually transmitted agents, HPVs are believed to be a contributing etiological factor in genital cancers including carcinoma of the uterine cervix. The association between HPV infection and cervical neoplasia has been reported to satisfy all accepted criteria for assessing casuality from epidemiologic studies (Kaufman et al. 1997; Villa 1997; Zehbe and Wilander 1997). Mucosal and genital HPVs, consisting of about 30 types, are divided into low risk (HPVs 6, 11, 42, 43, and 44) and high risk (HPVs 16, 18, 31, 33, 35, 45, 51, 52 and 56), according to their presence in malignant lesions of the cervix (Lowy et al. 1994). Recognized initially as sexually transmitted agents, HPVs are now considered human carcinogens (N Munoz 2000). Numerous epidemiological studies indicate a casual relationship between HPV infection and cervical neoplasia (Milde-Langosch et al. 2000). Functionally high-risk HPV infection contributes to carcinogenesis and tumor progression predominantly through the actions of two viral oncogenes, E6 and E7. These oncogenes are consistently expressed in cervical cell lines and in human cancers (Milde-Langosch et al. 2000, McMurray et al. 2001). Both of these oncogenes interact with and inhibit the activities of critical components of cell cycle regulatory systems, in particular E6 with p53 and E7 with Rb (MildeLangosch et al. 2000, McMurray et al. 2001, Philips et al. 1999). The E7 protein interacts with $\mathrm{pRB}$ and inactivates this cellular protein (Dyson et al. 1989). As a consequence, E2F transcription factor is released from $\mathrm{pRB}-\mathrm{E} 2 \mathrm{~F}$ complex, leading to transcriptional activation of several genes involved in cell proliferation (Sellers et al. 1997). Binding of the E6 protein to the p53 promotes the degradation of the latter through a ubiquitindependant proteolysis system. Also of significance is that on completion of the degradation of p53 by the ubiquitin-dependant proteolysis system, the E6 protein is free to interact again with remaining p53 molecules, leading to further degradation of the latter (Scheffner 1998). These events are summarized in Figure 1.

\section{HPV Variants}

The E6 and E7 genes have been studied in different patient populations and a number of variants have been described (Ginnoudis et al. 2001; Nindl et al. 1999; Xu et al. 2001; Van Duin et al. 2000). It can be classified into more than 40 variants and may be related to differences in progression of squamous intraepithelial lesions. The definition of an E6 variant is based on the departure from an original prototype first isolated from an invasive cervical carcinoma in Germany (Ginnoudis et al. 2001). These variants have also been geographically mapped to different areas of the world based on sequence variations of the E6, L1, L2, and long control regions (LCRs) (Yamada et al. 1997). Studies from our laboratory provided the first report on HPV 16 E6 and E7 gene variations from India and provide evidence for the association of specific E6 gene variants with the risk of cervical cancer. That these particular variants may contribute to increased risk and/or severity of cervical carcinoma is supported by in vitro studies demonstrating that differences exist in the immortalizing activity, transforming potential and rate/extent of p53 degradation of the different HPV 16 variants (Pillai et al. 2002).

\section{P53 Polymorphisms}

Genetic polymorphism is of significance in cancer because the polymorphic variants are thought to affect any one of the following features in disease progression, i.e., they possess variation in metabolism of carcinogens and drugs, alter risk from exposure, alter effectiveness of therapy, or variation may play a role in disease. While E6 variants may be important in contributing to increased severity of cervical cancer, polymorphisms of other host cellular proteins, such as p53 and p73, may also play an as yet undefined role in modulating the E6-mediated carcinogenic process (Zehbe et al. 2001; Park et al. 2001). P53 is a tumor suppressor gene that responds to genotoxic stress such as DNA damage and hypoxia and is considered the "guardian of the genome" (Lane 1992) and was designated "molecule of the year" in 1993 (Culotta and Koshland 1993). It maintains genomic integrity by arresting cell cycle progression or by inducing apoptosis (Sidransky and Hollstein 1996; Soussi and Beroud 2001). A p53 polymorphism in codon 72 has been described, which encodes either arginine or proline residues. Interestingly, the frequency of the arginine allele increases proportionally to the latitude, while the proline allele shows an inverse effect, i.e., it is more frequent in Black populations, and the arginine allele pre- 


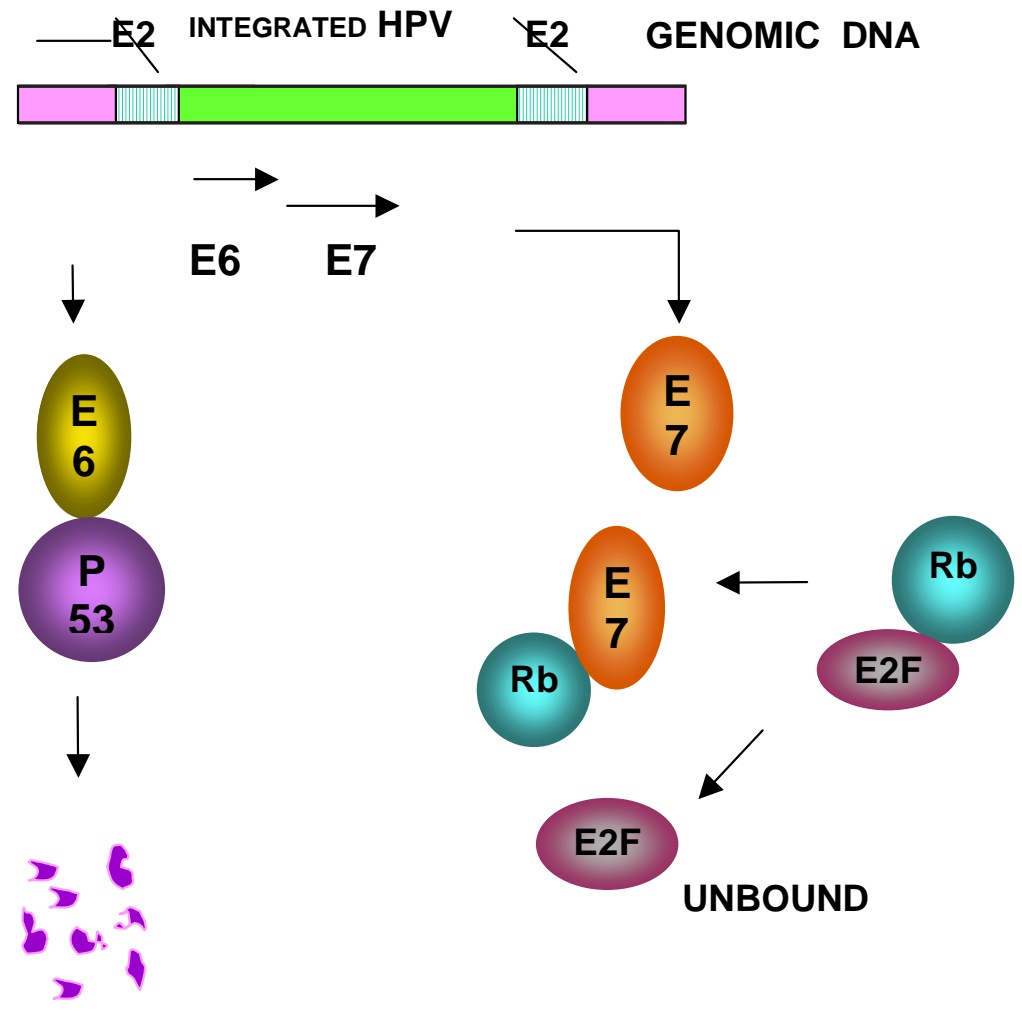

\section{LOSS OF TUMOR}

SUPPRESSION

GENE TRANSCRIPTION
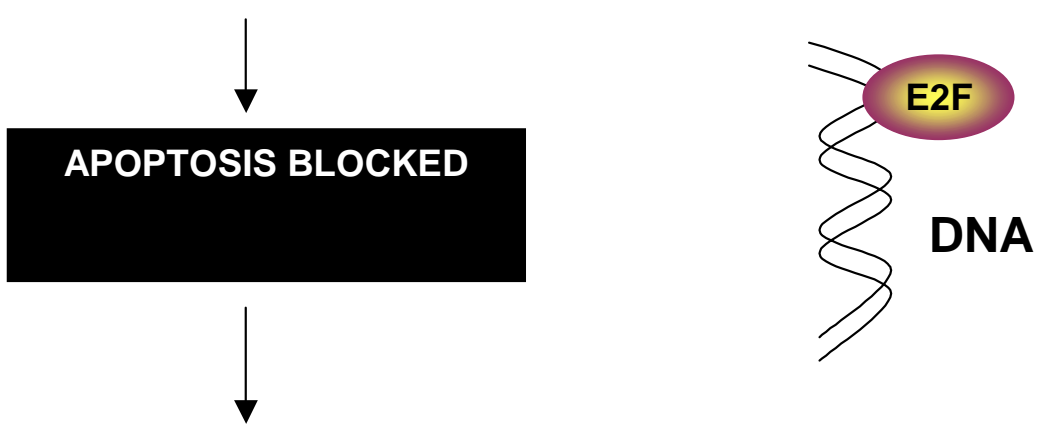

ONCOGENESIS

Fig. 1. HPV and Cancer

CELL CyCLE

ACTIVATION;

PROLIFERATION 
dominates among Caucasians (Beckman et al. 1994). It has been observed that the p53 arginine variant is more susceptible to HPV E6 mediated degradation than the proline variant. Individuals homozygous for the arginine allele had a 7 times higher chance to bear an HPV associated SCC of the cervix than heterozygous proline/arginine women (Storey et al. 1998). This sensitivity of the Arginine variant could thus be clinically important, as it has been clearly established that p53 degradation is an important feature of HPV associated tumors. However, subsequent analyses performed in the similar and different populations were contradictory making the issue controversial (Rosenthal et al. 1998; Giannoudis et al. 1999; Minaguchi et al. 1998). The two previous pilot studies from India were also contradictory. One report from Western India showed evidence for increased risk of Arginine variant (Saranath et al. 1999), while the other report from Northern India found no evidence for increased risk (Das et al. 1999). Our study, which included various stages of pre-malignant cervical lesions found no evidence of increased risk for progression associated with Arginine homozygosity in any of these subgroups (Pillai et al. 2002).

\section{Tobacco}

Tobacco smoking (either active or passive) and chewing has also been found to be associated with increased risk of cervical malignancy (Parazzini et al. 1998; Daling et al. 1996). Nicotine, cotinine and tobacco specific nitosamines have been detected in cervical mucus of smokers and those exposed to tobacco smoke (Prokopczyk et al. 1997; McCann et al. 1992). DNA adducts of aromatic compounds, have been reported to be found with increased frequency in the cervical epithelium of smokers compared to non-smokers (Simons et al. 1993). Reports also showed that women with exposure to wood smoke had a higher risk of cervical neoplasia (Velema et al 2002). Smoking may predispose a woman to the development of cervical cancer by lowering the immune surveillance at the cellular level also (Prokopczyk et al. 1997; Mc Cann et al. 1992). Tobacco use is causally associated with cancers of the lung, larynx, mouth, esophagus, kidneys, urinary tract, and possibly, breast. Major classes of carcinogens present in tobacco and tobacco smoke are converted into DNA-reactive metabolites by cytochrome P450 (CYP)-related enzymes, several of which display genetic polymorphism. Individual susceptibility to cancer is likely to be modified by the genotype for enzymes involved in the activation or detoxification of carcinogens in tobacco and repair of DNA damage.

The mechanism by which PAHs such as $\mathrm{B}[a] \mathrm{P}$ interact with DNA, activate oncogenes, and initiate the carcinogenic process involves the formation of bay-region diolepoxides as the major ultimate carcinogens. $\mathrm{B}[a] \mathrm{P}$ is converted into phenolic metabolites and $\mathrm{B}[a] \mathrm{P}-7,8$-diol by a CYP-mediated process. Secondary metabolism, mainly involving epoxide hydrolase and other CYP isoforms, leads to the formation of the highly reactive (+)-anti-BPDE. Several carcinogens present in tobacco smoke are inactivated by GSTs. The most frequently studied carcinogenic PAH diolepoxide, BPDE, is a relatively good substrate for GSTM1, M2, and M3 and better still for GSTP1 (Bartsch et al. 2000). Because tobacco carcinogens, ROS, and lipid peroxidation products are likely to be substrates for GSTT1 or $M 1$, the extent of DNA damage and ultimately the cancer risk may be affected by polymorphic CYPs and GST detoxifying enzymes (Brockmoller et al. 1998; Nair et al. 1999).

\section{Xenobiotic Metabolizing Enzyme (XME) Gene Polymorphisms}

Polymorphisms of genes involved in metabolism of various endogenous and exogenous carcinogens are relatively common in most populations. Generally carcinogens are oxidized to reactive intermediates by phase I enzymes (eg. CYPs), while phase II enzymes like glutathione S-transferases (GST) generally mediate the conjugation of water soluble moieties (such as glutathione) to these reactive metabolites, rendering them harmless (Miller et al. 2001).

P450 cytochromes (CYP) are enzymes, which catalyze the insertion of one atom of molecular oxygen into a substrate. This is a typical reaction of activation (phase I) which converts indirect carcinogens into active electrophiles capable of interacting with the biological macromolecules DNA, RNA and proteins. CYP are coded by genes of the CYP super family (Pavanello et al. 2000). Glutathione S-transferases(GSTs) are one of the major groups of detoxifying enzymes. Each GST has distinct catalytic properties: conjugation with glutathione, peroxidation and isomerization. The cytosolic GSTs known until now belong to five 
different classes, are coded by atleast five gene families and according to their primary amino acid sequence, are called GST, classes $\alpha, \mu, \pi, \sigma$ and $\theta$ (Hayes et al. 1995).

Reactive metabolites that are not detoxified may react with DNA to form DNA adducts which, if not required, may eventually produce somatic mutations and cancer. Of great interest in the study of xenobiotic metabolism is the existence of polymorphisms in animal model systems and humans in which a large percentage of the alleles of a particular gene are inactive (Gonzalez et al. 1994). Polymorphisms have been found in the P450s and many phase II enzymes. In humans, P450 polymorphisms are known to affect drug therapy. Polymorphisms in the carcinogenmetabolizing enzymes are thought to play a role in cancer susceptibility in humans. Associations of polymorphisms with cancer risk will be especially important in cases where there are known exposures to chemical carcinogens such as with tobacco smoking, high intake of food mutagens and industrial exposures (Caporaso et al. 1995). It has been reported that HPV infection, through the modulation of cellular xenobiotic metabolizing enzymes, may play a role in the ability of cells to handle environmental carcinogens (Chen et al. 1999). Two genetic polymorphisms of the CYP1A1 gene have been reported to be associated with differences in the activity of the enzyme aryl-hydrocarbon hydroxylase (AHH) activity (H Autrup 2000) - an isoleucine to valine substitution in exon 7 at the $\mathrm{Nco}$ I restriction site (m2 polymorphism) and a thymine/cytosine point mutation in the Msp1 restriction site ( $\mathrm{m} 1$ polymorphism). The $\mathrm{m} 2$ (valine variant) displays a two fold higher catalytic activity compared to the wild type enzyme (H Autrup 2000). The significance of these polymorphisms in carcinogenesis is still unclear. The homozygous $\mathrm{m} 2$ polymorphism has been shown to strongly correlate to lung cancer incidence among Japanese although such dramatic results were not obtained for Caucasians (Merchand et al. 1998). We had earlier reported increased frequency of the $\mathrm{m} 2$ polymorphism in oral cancer patients with a long history of tobacco use (T T Sreelekha et al. 2001). Two previous studies on cervical cancer have shown the importance of CYP1A1 polymorphisms. Women from Hawaii who were homozygous for the CYP1A1 Msp variant allele (m1) had an odds ratio of 3.4 of having cervical intraepithelial lesions compared to women homozygous for the wild allele (Goodman et al. 2001). However Kim and colleagues did not find this association in Korean women (Kim et al. 2000). Women with $\mathrm{m} 1$ and $\mathrm{m} 2$ CYP1A1 polymorphisms and with prolonged exposure to firewood smoke, tobacco smoke or tobacco products will therefore have higher levels of reactive metabolites capable of causing DNA damage, in addition to a preexisting HPV infection. Studies conducted from our laboratory show that subjects who were HPV 16 positive had an odds ratio of $3.0(95 \%$ Confidence Interval $=1.8$ to 4.8$)$ and $2.9(95 \%$ Confidence Interval $=1.8$ to 4.6 ) of having a $\mathrm{m} 1$ and $\mathrm{m} 2$ polymorphism respectively (Pillai 2004, data submitted). A recent report has provided epidemiological evidence for the significant increased risk of cervical carcinoma in HPV infected women having prolonged exposure to firewood smoke (Velema et al. 2002). We have also observed that deletion of both GSTM1 and GSTT1 was significant in cases compared to controls. Unlike in the case of the CYP1A1 m1 and $\mathrm{m} 2$ variants, there was a moderate risk of GSTM1 deletion in relation to age (Odds Ratio = $1.8,95 \%$ Confidence Interval $=1.17$ to 2.77 ). No such association was evident in the case of GSTT1. There was also an elevated risk for women who were HPV positive of having GSTM1 and GSTT1 deletions (Odds Ratio $=1.6,95 \%$ Confidence Interval $=1.1$ to 2.5 for GSTM1 and Odds Ratio $=1.7,95 \%$ Confidence Interval $=0.9$ to 2.9).

\section{CONCLUSION}

It is obvious therefore that the genetic polymorphisms in metabolic enzymes may play a role in development of cervical cancer and more importantly may act as a co-factor in HPV associated carcinogenesis. Cancer occurring in HPV infected tissue can take fifteen to twenty years to develop. During this intervening period, increased chemical carcinogen exposure coupled with inefficient clearing owing to genetic polymorphisms of xenobiotic metabolizing enzymes can be a significant factor. In India, which has a high incidence of HPV associated cancer, as well as in many developing countries the disease is associated with poor socioeconomic conditions. Such women are often exposed to a wide range of carcinogens including those derived from tobacco use, prolonged and sustained inhalation of smoke from kitchen firewood use and possibly 
the increased exposure to pesticides from working in the agricultural sector. It is thus clear that large population studies are required in specific populations to determine the biological properties of natural E6 gene variants and eventually to define the molecular mechanisms involved in HPV oncogenecity.

\section{REFERENCES}

Autrup H 2000. Genetic polymorphisms in human xenobiotica metabolizing enzymes as susceptibility factors in toxic response. Mutation Res, 464: 6576.

Bartsch H, Nair U, Risch A, Rojas M, Wikman H, and Alexandrov K 2000. Genetic polymorphism of CYP genes, Alone or in Combination, as a Risk Modifier of Tobacco-related Cancers. Cancer Epidemiology Biomarkers and Prevention, 9: 3-28.

Beckman G, Birgander R, Sjalander A, Saha N, Homberg PA, Kivela A and Beckman L 1994. Is p53 polymorphism maintained by natural selection? Human Heredity, 44: 266-270.

Bosch F X, Munoz N 2002. The viral etiology of cervical cancer. Virus Research, 89: 183-190.

Bosch F.X, Lorincz A, Munoz N, Meijer C J L M, Shah K V 2002. The casual relation between human papilloma virus and cervical cancer. J Clin Pathol, 55: $244-265$.

Brockmoller J, Cascorbi I, Kerb R, Sachse C, Roots I 1998. Polymorphisms in xenobiotic conjugation and disease predisposition. Toxicol Lett, 102: 173183.

Caporaso N, Goldstein A 1995. Cancer genes: single and susceptibility-exposing the difference. Pharmacogenetics, 5: 59-63.

Chen C and Nirunsuksiri W 1999. Decreased expression of glutathione s-transferase M1 in HPV16transfected human cervical keratinocytes in culture. Carcinogenesis, 20-4: 699-703.

Culotta and Koshland 1993. P53 sweeps through cancer research. Science, 262: 1958-1961.

Daling JR, Madeleine MM, McKnight B, Carter JJ, Wipf GC, et al 1996. The relationship of human papillomavirus related cervical tumors to cigarette smoking, oral contraceptive use, and prior Herpes Simplex virus type 2 infection. Cancer Epidemiol Biomarkers Prev, 5: 541-548.

Das BC, Katiyar S, Thelma BK 1999. P53 tumor suppressor gene and human cancers. Transcriptional control of HPV oncogene expression. 18 ${ }^{\text {th }}$ Annual convention of Indian Association for Cancer Research and National Symposium on Molecular Biology of Cancer, AIIMS, New Delhi, India. Abstract Book; pp 40.

Dyson N, Howley PM, Munger K and Harlow E 1989. The HPV16 E7 oncoprotein is able to bind to the retinoblastoma gene product. Science, 242: 934937.

Ginnoudis A, Herrington CS 2001. Human papillomavirus variants and squamous neoplasia of the cervix. J Pathol, 193: 295-302.

Gonzalez FJ, Idle JR 1994. Pharmacogenetic phenotyping and genotyping. Present status and future potential. Clin Pharmacokinet, 26: 59-70.

Goodman MT, Mc Duffie K, Hernandez B, Berttram CC, Wilkens LR, et al. 2001. CYP1A1, GSTM1 and GSTT1 polymorphisms and the risk of cervical squamous intraepithelial lesions in a multiethnic population. Gynecol Oncol, 81: 263-269.

Hayes JD, Pulford DJ 1995. The glutathione s-transferase supergene Family Regulation of GST and the contribution of the isoenzymes to cancer chemoprotection and drug resistance, Crit Rev Biochem Mol Biol, 30: 445-600.

Jayant K, Rao RS, Nene BM 1995. Improved stage at diagnosis of cervical cancer with increased cancer awareness in a rural Indian population. Int J Cancer, 63: $161-3$.

Kaufman R H, Adam E, Kenogle J, et al 1997. Relevance of Human Papilloma Virus screening in the management of cervical intraepithelial neoplasia. Am J Obstet Gynecol, 176: 87-92.

Kim JW, Lee CG, Park YG, Kim KS, Kim IK, et al. 2000. Combined analysis of germline polymorphisms of p53, GSTM1, GSTT1, CYP1A1, and CYP2E1: relation to the incidence rate of cervical carcinoma. Cancer, 88: 2082-2091.

Lane DP 1992. P53, guardian of the genome. Nature, 358: $15-16$.

Lowy DR, Kimbauer R, and Schiller JT 1994. Genital human papillomaviruses. Proceedings of the National Academy of Sciences USA, 91: 24362440.

Marchand LL, Sigfried A, Lum A, Wilkins LR, Lau AF 1998. Association of CYP1A1, GSTM1 and CYP2E1 polymorphisms with lung cancer suggest cell type specifications to tobacco carcinogens. Cancer Res, 58: 4858-4863.

McCann MF, Irwin DE, Walton LA, Hulka BS, Morton JL, et al 1992. Nicotine and cotinine in the cervical mucus of smokers, passive smokers, non-smokers, Cancer Epidemiol Biomarkers Prev, 1: 125-129.

McMurray HR, Nguyen D, Westbrook TF, McCance DJ 2001. Biology of human papillomaviruses. Int $J$ Exp Pathol, 82: 15-33.

Milde-Langosch K, Reithdorf S, Loning T 2000. Association of human papillomavirus infection with carcinoma of the cervix uteri and its precursor lesions: theoretical and practical implications. Virchows Arch, 437: 227-33.

Miller MC, Mohrenweiser HW, Bell DA 2001. Genetic variability in susceptibility and response to toxicants. Toxicol Lett, 120: 269-280.

Munoz N 2000. Human papillomavirus and cancer: the epidemiological evidence. J Clin Virol, 19: 1-5.

Nair UJ, Nair J, Mathew B, Bartsch H 1999. Glutathione $\mathrm{S}$-transferase $\mathrm{M} 1$ and $\mathrm{T} 1$ null genetypes as risk factors for oral leukopakia in ethnic Indian betel quid/tobacco chewers. Carcinogenesis (Lond.), 5: 743-748

Nindl I, Rindfleisch K, Lotz B, Schneider A, Durst M 1999. Uniform distribution of HPV 16 E6 and E7 variants in patients with normal histology, cervical intraepithelial neoplasia and cervical cancer. Int $J$ Cancer, 82: 203-207.

Parazzini F, Chatenoud L, La Vecchia C, Negri E, Franceschi S, et al 1998. Determinants of risk of 
invasive cervical cancer in young women. $\mathrm{Br} J$ Cancer, 77: 834-841.

Park JS, Kim EJ, Lee JY, Sin HS, Namkoong SE, et al 2001. Functional inactivation of p73, a homolog of p53 tumor suppressor protein by human papillomavirus E6 proteins. Int J Cancer, 91: 822827.

Pavanello S, Clonfero E 2000. Biological indicators of genotoxic risk and metabolic polymorphisms. Mutation Research, 463: 285-308.

Philips AC, Voudsen KH 1999. Human papillomavirus and cancer: the viral transforming genes. Cancer Surv, 33: 55-74.

Pillai MR, Sreevidya S, Pollock BH, Jayaprakash PG, Herman B 2002. Polymorphism at codon 72 of p53, human papillomavirus and cervical cancer in South India. Journal of Cancer Research and Clinical Oncology, 128: 627-631.

Prokopczyk B, Cox JE, Hooffmann D, Waggoner SE 1997. Identification of tobacco specific carcinogen in the cervical mucus of smokers and nonsmokers. J Natl Cancer Inst, 89: 868-873.

Saranath D, Khan Z, Shrivastava SK, Kane S, Amin G, et al 1999. Prevalence of HPV16/18 and p53 polymorphisms in cervical cancer in Indian women. Proceedings of the Sixth Annual Ranbaxy Science Foundation Symposium on Recent Developments in the Management of Cervical Cancer, Ranbaxy Science Foundation, New Delhi pp 57-59.

Scheffner M 1998. Ubiquitin, E6-AP and their role in p53 inactivation. Pharmacol Ther, 78: 129-139.

Sellers WR and Kaelin Jr, WG 1997. Role of Rb protein in the pathogenesis of human cancer. Journal of Clinical Oncology, 15: 3301-3312.

Sidransky D, Hollstein M 1996. Clinical implications of the p53 gene. Annu Rev Med, 47: 285-301.

Simons AM, Philips DH, Coleman DV 1993. Damage to DNA in cervical epithelium related to smoking tobacco. Br Med J, 306: 1444-1448.

Soussi T, Beroud C 2001. Assessing TP53 status in human tumours to evaluate clinical outcome. Nature Rev (Cancer), 1: 233-240.
Sreelekha TT, Ramdas K, Pandey M, Thomas G, Nalinakumari KR, Pillai MR 2001. Genetic polymorphism of CYP1A1, GSTM1 and GSTT1 genes in Indian oral cancer. Oral Oncology, 37: 593-598.

Storey A, Thomas M, Kalita A, Harwood C, Gardiol D, Mantovani F, Breuer J, Leigh IM, Matlashewski G and Banks L 1998. Role of a p53 polymorphism in the development of HPV- associated cancer. Nature, 393: 229-234.

Van Duin M, Snijders PJF, Vossen MTM, Klaassen E, Voorhost F, et al 2000. Analysis of human papillomavirus $16 \mathrm{E} 6$ variants in relation to p53 codon 72 polymorphism genotypes in cervical carcinogenisis. J Gen Virol, 81: 317-325.

Velema JP, Ferrera A, Figueroa M, Bulnes R, Toro LA, et al 2002. Burning wood in the kitchen increases the risk of cervical neoplasia in HPV-infected women in Honduras. Int J Cancer, 97: 536-541.

Villa LL 1997. Human papillomaviruses and cervical cancer. Adv. Cancer Res., 71: 321-341.

Xu X, Pang T, Guo Z, Mazurenko N, Kisselijov F, et al 2001. HPV 16 E6 gene variations in invasive cervical carcinoma and cancer in situ from Russian patients. $\mathrm{Br}$ J Cancer, 84: 791-795.

Yamada T, Manos MM, Peto J, Greer CE, Munoz N, et al 1997. Human papilloma type 16 sequence variation in cervical cancers: a worldwide perspective. J Virol, 71: 2463-2472.

Zehbe I, Voglino G, Wilander E, Delius H, Marongiu A et al 2001. P53 codon 72 polymorphism and various human papillomavirus 16 E6 genotypes are risk factors for cervical cancer development. Cancer Res, 61: 608-611.

Zehbe I, and Wilander E 1997. Human papillomavirus infection and invasive cervical neoplasia: A study of prevalence and morphology. J Pathol, 181: 270275 .

Zur Hausen H 1996. Papilloma virus infections - a major cause of human cancers. Biochemica et Biophysica Acta, 1288: F55-F78. 